This is an electronic reprint of the original article. This reprint may differ from the original in pagination and typographic detail.

\author{
Author(s): Vormawah, L. J.; Vilén, Markus; Beerwerth, R.; Campbell, P.; Cheal, B.; Dicker, A.; \\ Eronen, Tommi; Fritzsche, S.; Geldhof, Sarina; Jokinen, Ari; Kelly, S.; Moore, Iain; \\ Reponen, Mikael; Rinta-Antila, Sami; Stock, S. O.; Voss, Annika
}

Title: Isotope shifts from collinear laser spectroscopy of doubly charged yttrium isotopes

Year: $\quad 2018$

Version:

Please cite the original version:

Vormawah, L. J., Vilén, M., Beerwerth, R., Campbell, P., Cheal, B., Dicker, A., Eronen, T., Fritzsche, S., Geldhof, S., Jokinen, A., Kelly, S., Moore, I., Reponen, M., RintaAntila, S., Stock, S. O., \& Voss, A. (2018). Isotope shifts from collinear laser spectroscopy of doubly charged yttrium isotopes. Physical Review A, 97(4), Article 042504. https://doi.org/10.1103/PhysRevA.97.042504

All material supplied via JYX is protected by copyright and other intellectual property rights, and duplication or sale of all or part of any of the repository collections is not permitted, except that material may be duplicated by you for your research use or educational purposes in electronic or print form. You must obtain permission for any other use. Electronic or print copies may not be offered, whether for sale or otherwise to anyone who is not an authorised user. 


\title{
Isotope shifts from collinear laser spectroscopy of doubly charged yttrium isotopes
}

\author{
L. J. Vormawah, ${ }^{1}$ M. Vilén, ${ }^{2}$ R. Beerwerth,,${ }^{3,4}$ P. Campbell, ${ }^{5}$ B. Cheal, ${ }^{1, *}$ A. Dicker, ${ }^{5}$ T. Eronen, ${ }^{2}$ S. Fritzsche,,${ }^{3,4}$ S. Geldhof, ${ }^{2}$ \\ A. Jokinen, ${ }^{2}$ S. Kelly, ${ }^{5}$ I. D. Moore, ${ }^{2}$ M. Reponen, ${ }^{2}$ S. Rinta-Antila, ${ }^{2}$ S. O. Stock, ${ }^{3,4}$ and A. Voss ${ }^{2}$ \\ ${ }^{1}$ Department of Physics, University of Liverpool, Liverpool L69 7ZE, United Kingdom \\ ${ }^{2}$ Department of Physics, University of Jyväskylä, PB 35(YFL) FIN-40351 Jyväskylä, Finland \\ ${ }^{3}$ Helmholtz Institut Jena, Fröbelstieg 3, 07743 Jena, Germany \\ ${ }^{4}$ Theoretisch-Physikalisches Institut, Friedrich-Schiller-Universität Jena, 07743 Jena, Germany \\ ${ }^{5}$ School of Physics and Astronomy, University of Manchester, Manchester M13 9PL, United Kingdom
}

(Received 4 February 2018; published 16 April 2018)

\begin{abstract}
Collinear laser spectroscopy has been performed on doubly charged ions of radioactive yttrium in order to study the isotope shifts of the 294.6-nm $5 s^{2} S_{1 / 2} \rightarrow 5 p^{2} P_{1 / 2}$ line. The potential of such an alkali-metal-like transition to improve the reliability of atomic-field-shift and mass-shift factor calculations, and hence the extraction of nuclear mean-square radii, is discussed. Production of yttrium ion beams for such studies is available at the IGISOL IV Accelerator Laboratory, Jyväskylä, Finland. This newly recommissioned facility is described here in relation to the on-line study of accelerator-produced short-lived isotopes using collinear laser spectroscopy and application of the technique to doubly charged ions.
\end{abstract}

DOI: 10.1103/PhysRevA.97.042504

\section{INTRODUCTION}

High-resolution optical spectroscopy is of fundamental importance to nuclear structure research and must be applied to short-lived radioactive isotopes to understand how nuclear properties change with proton and neutron number [1-3]. These isotopes are produced at specialist "isotope factories" where typically a high-energy beam of charged particles from an accelerator is impinged on a sample of target material in order to induce nuclear reactions. The products are then extracted in the form of a low-energy $(30-60 \mathrm{keV})$ beam of ions, manipulated and transported to experimental stations. The most common high-resolution form of such spectroscopy is collinear laser spectroscopy, in which a laser beam and a radioactive ion beam are overlapped in a parallel geometry. Collinear laser spectroscopy is used at many different facilities; examples include ISOLDE (CERN, Switzerland) [4], JYFL (Jyväskylä, Finland) [5], NSCL (Michigan, USA) [6], and TRIUMF (Vancouver, Canada) [7], with complementary techniques for heavy elements [8,9]. For this work, beams of neutron-rich isotopes of yttrium are uniquely available at the JYFL IGISOL IV facility.

The mean-square charge radius of a nucleus is a quantity of key importance for studies of nuclear sizes and shapes, and can be extracted from isotope shift measurements in a nuclear model-independent way. The change in nuclear mean-square charge radius, $\delta\left\langle r^{2}\right\rangle^{A, A^{\prime}}$, from isotopes $A$ to $A^{\prime}$, is related to the isotope shift, $\delta v^{A, A^{\prime}}$, by [1]

$$
\delta v^{A, A^{\prime}}=M \frac{m_{A^{\prime}}-m_{A}}{m_{A} m_{A^{\prime}}}+F \delta\left\langle r^{2}\right\rangle^{A, A^{\prime}},
$$

where $m$ is the atomic mass [10], and $M$ and $F$ are the respective atomic factors for the mass shift and field shift. Thus,

\footnotetext{
*bradley.cheal@liverpool.ac.uk
}

these two constants need to be evaluated in order to extract the values of $\delta\left\langle r^{2}\right\rangle$ for a chain of isotopes. Normally, $F$ and $M$ are calibrated through nonoptical isotope shift data, obtained using methods such as $K$ x-ray spectroscopy, muonic atom spectroscopy, or electron scattering. These methods require a macroscopic sample and are therefore limited to stable or long-lived isotopes. Additionally, optical isotope shifts may have been measurable (at low efficiency) using a vaporized sample for a variety of transitions, easing the atomic calculations or providing a crosscheck. Such measurements are then incorporated into a combined analysis of radii reference data $[11,12]$, suitable for use as a calibration, providing at least three stable isotopes are present. A new facility at RIKEN, Japan, aims to apply electron scattering to radioactive ion beams [13], but production may not be versatile across the periodic table, and elements such as yttrium may be problematic, as they are for conventional isotope separation on-line (ISOL) facilities, traditionally used for collinear laser spectroscopy.

In the $Z \approx 40$ region of the nuclear chart, a sudden onset of nuclear deformation occurs at $N \approx 60$ [5,14-16]. This dramatic shape transition is accompanied by a sharp increase in mean-square nuclear charge radius. The yttrium isotope chain is of particular interest, as it appears to fall right in the center of this deformed region of the nuclear chart and is seemingly the element for which the effect is most prominent [14]. From $N=50$ to $N=59$, the yttrium nuclei were observed to become increasingly oblate, accompanied by a proportional increase in nuclear softness, having a significant dynamic component to the total deformation. At $N=60$, this appears to change abruptly to a rigidly deformed prolate shape. A measure of the nuclear softness is given by the difference between experimental charge radii and static-only estimates calculated using the nuclear electric quadrupole moments. Therefore a reliable calibration of $F$ and $M$ is important in drawing conclusions on nuclear softness and in comparing the nuclear charge radii with those of neighboring element isotope chains. 
The main issue for reliably determining the mean-square charge radii is that yttrium has only one stable isotope, ${ }^{89} \mathrm{Y}$. Nonoptical data for changes in charge radii along the yttrium chain, to which calibration could be attempted, are not available. One must therefore rely on multiconfiguration Dirac-Fock (MCDF) calculations, or semiempirical estimates, of both $F$ and $M$. Similar problems have recently arisen in studies of niobium [5], scandium [17] and manganese [18,19], and, to a lesser extent, also copper [20] and gallium [21], which each have two stable isotopes. In these latter cases, (nuclear model dependent) nonoptical data could at least be used to correct the mass shift factor, which is the more challenging factor of the two to calculate due to the stronger dependence on electron correlations.

The MCDF method allows atoms with many open shells to be treated in a similar manner to closed-shell atoms. An atomic energy level $\alpha$ is represented by a sum of configuration state functions (CSFs) [22],

$$
\phi_{\alpha}(P J M)=\sum_{r=1}^{n_{c}} c_{r}(\alpha)\left|\gamma_{r} P J M\right\rangle,
$$

all of which exhibit the same symmetry. Here, $n_{c}$ is the number of CSFs present, and $c_{r}(\alpha)$ represents the level in this basis. A spherical nucleus is assumed in order to obtain a single field-shift factor $F$ for a whole isotope chain. In order to do this, a Fermi distribution is assumed to hold for the nuclear charge density [22]

$$
\rho(r)=\frac{\rho_{0}}{1+e^{(r-c) / a}},
$$

where $c$ is the radius at half-charge density, and $a$ is related to the nuclear skin depth $t$ by

$$
a=\frac{t}{4 \ln 3} .
$$

For more complex transitions involving many electrons, difficulties in correctly calculating the $F$ and $M$ factors often arise from missed correlation contributions. While the MCDF method takes this into account by incorporating electron "replacements" from bound states to virtual "active" states, the rapid increase in the size of the wave function requires truncation of the active space. For this reason, this approach has mainly been tested for isotope shifts of lighter elements and isotopes with simpler valence-shell structures [22].

For yttrium, laser spectroscopy was previously performed on singly charged ions of ${ }^{86-90,92-102} \mathrm{Y}$, using the $363.3-\mathrm{nm}$ $5 s^{2}{ }^{1} S_{0} \rightarrow 4 d 5 p^{1} P_{1}$ transition [14]. Although exhibiting a high spectroscopic efficiency, an evaluation of the $F$ and $M$ factors could only be performed empirically, by assuming radial trends similar to other isotopes in the region. This can be ambiguous, because in regions rich in nuclear structural change, it is difficult to know which (if any) of the isotopes are expected to display "normal" behavior and be suitable for use as anchor points. Later, values of $F$ and $M$ were calculated using the MCDF method for two different transitions in the $\mathrm{Y}^{+}$ ion. These were the aforementioned 363.3-nm transition and also the $321.7-\mathrm{nm} 4 d 5 s^{3} D_{2} \rightarrow 4 d 5 p^{3} P_{1}$ transition, where selected isotopes have been remeasured, ostensibly to determine the nuclear spin of ${ }^{100 \mathrm{~m}} \mathrm{Y}$ [23]. The calculations performed yielded inconsistent values of $\delta\left\langle r^{2}\right\rangle$, scattering to either side of the semiempirical estimate [22]. Although having isotope shift measurements for two transitions enabled an assessment of consistency by extracting radii from both lines for a common set of isotopes or analysis via a King plot, the transitions were selected on the basis of experimental considerations alone.

Usually, atomic transitions are selected for experimental study on the grounds of their spectroscopic efficiency and sensitivity. Collinear laser spectroscopy typically has the capability to access exotic isotopes which have ion currents from around $100 \mathrm{~s}^{-1}$ [24]. However, in principle it is possible to identify transitions where MCDF calculations are easier or more reliable. While such transitions may have a lower spectroscopic efficiency, only three isotopes (two isotope shifts) need to be remeasured, and this is sufficient to recalibrate the charge radii measurements for the whole isotope chain. The 294.6-nm $5 s^{2} S_{1 / 2} \rightarrow 5 p^{2} P_{1 / 2}$ line in the $\mathrm{Y}^{2+}$ ion [25] promised a simpler atomic structure and was therefore chosen for study in this work.

\section{EXPERIMENTAL METHODOLOGY}

Laser spectroscopy was performed at the IGISOL IV facility [26-28], University of Jyväskylä, Finland (Fig. 1). At the IGISOL, nuclear reactions are induced by impinging a beam of protons, deuterons, or alpha particles from one of two cyclotrons onto a foil of target material. These are a new MCC30 cyclotron, able to deliver protons at $30 \mathrm{MeV}$ and deuterons at $15 \mathrm{MeV}$, and a K130 cyclotron, which delivers also higher beam energies and heavier ions. Beam delivery from the $\mathrm{K} 130$ is, however, limited to beam currents of $<10 \mu \mathrm{A}$, whereas significantly higher currents will be available from the MCC30, and it is dedicated for IGISOL use. Reaction products recoil into an inert buffer gas and are subsequently carried out in a supersonic gas jet. This allows for the study of a range of radionuclides which are not available at facilities where conventional thick targets are used (due to the physical or chemical properties of the products $[29,30])$. Various "ion guide" gas cell designs are available for fission, light-ion induced fusion evaporation and heavy-ion reactions, sputtering of macroscopic samples, and laser resonance ionization.

\section{A. Production of radioactive yttrium ions}

Radioactive yttrium isotopes were produced via a protoninduced fission reaction with a $30-\mathrm{MeV}$ proton beam impinging on a thin foil of natural uranium or thorium. The reaction products recoil out of the target into a gas cell filled with 150-300 mbar of helium before being transported through a 1 -mm-diameter exit nozzle in a supersonic gas jet. Most of the charged ionic products leave the target chamber in the singly charged state; however, a small fraction $(\sim 10 \%)$ leave the IGISOL in a doubly charged state. This smaller (doubly charged) fraction was used for laser spectroscopy.

Ions were subsequently captured by a sextupole ion guide (SPIG) [31], consisting of six cylindrical rods with rf voltages applied to adjacent rods in antiphase. This focused the ions along the central axis towards a conical extraction electrode while high-throughput vacuum pumps were used to remove the neutral helium buffer gas and maintain the vacuum in the 


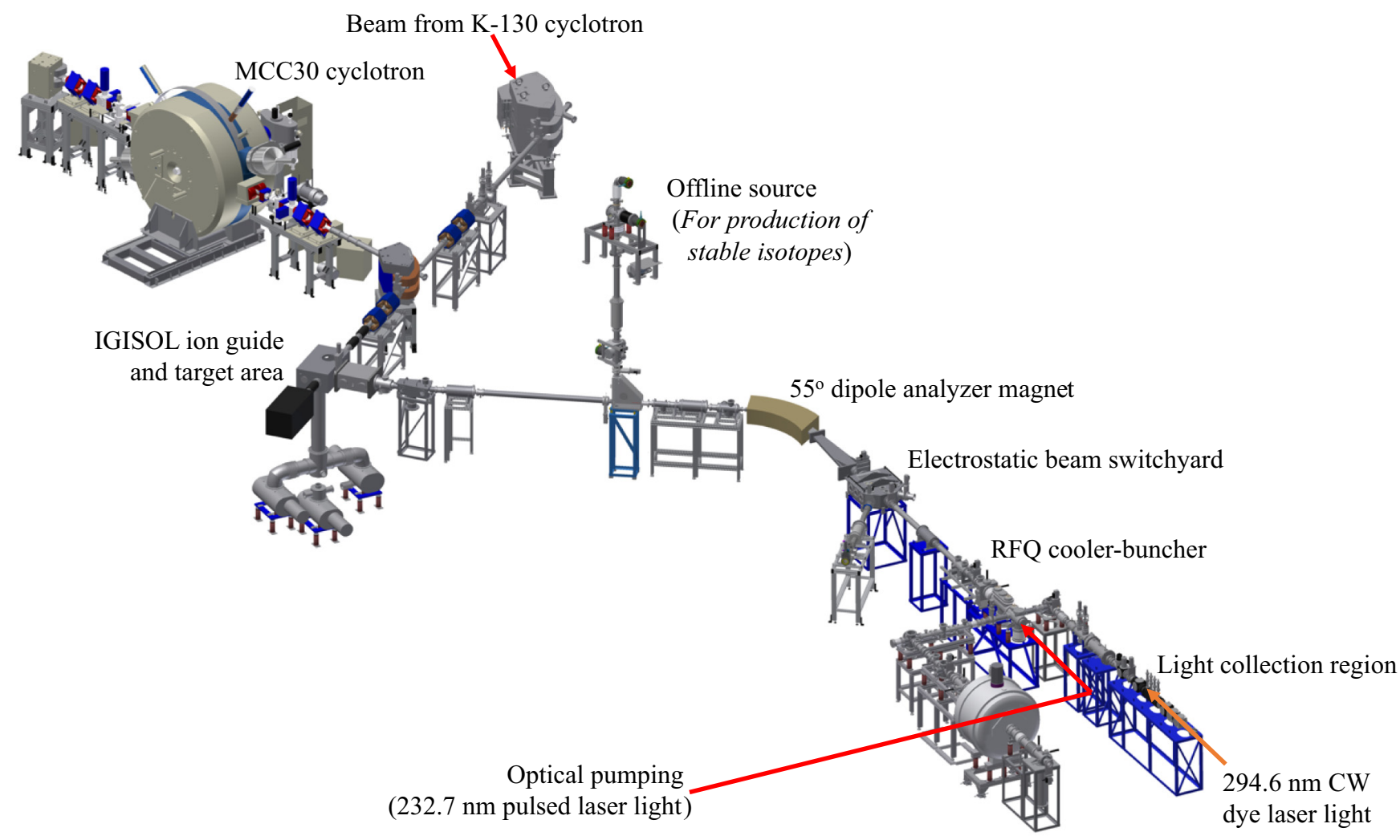

FIG. 1. The layout of the IGISOL IV facility, University of Jyväskylä, Finland. Radioactive $\mathrm{Y}^{2+}$ ions were produced from proton-induced fission reactions, formed into a beam and directed along the beamline to the laser spectroscopy station. To provide a frequency reference, stable ${ }^{89} \mathrm{Y}^{2+}$ ions were formed into a beam in a similar way but produced using the dedicated off-line source, located on the second floor of the facility.

outer chamber of the IGISOL. The resulting beam of ions was then accelerated via a $30-\mathrm{kV}$ potential applied to the IGISOL to an energy of $60 \mathrm{keV}$ due to the desired ions being in the doubly charged state, directed along the beamline and mass separated in-flight by means of passage through a magnetic dipole field.

\section{B. The RFQ cooler-buncher}

After mass separation, the ions were directed to the coolerbuncher, a rf quadrupole filled with a low-pressure (0.1 mbar) helium buffer gas $[15,32]$, placed on a $30-\mathrm{kV}$ high-voltage platform. The ions enter the cooler and collide with the helium atoms, losing a fraction of their kinetic energy upon each collision until all ions are left with a thermal kinetic energy of $<1 \mathrm{eV}$. A weak axial field guides the ions to the extraction end of the cooler, where a trapping potential is applied to accumulate the ions, typically for $200 \mathrm{~ms}$, before releasing them as a bunched beam of $10-\mu$ s temporal length. After release from the cooler, the ions are reaccelerated with a reduced emittance and longitudinal energy spread.

\section{In-cooler optical pumping}

Collinear laser spectroscopy of ions has traditionally been limited to the study of transitions from the ground state or very low-lying metastable states where the natural electronic population is significant. More recently, optical pumping has been used to enhance the population of a selected metastable state, from which collinear laser spectroscopy can be performed. Due to the long exposure time to the laser, efficient optical pumping can be achieved within the cooler-buncher [5], where optical access to the central axis is permitted by a twin quadrupole bend arrangement. Pulsed, broadband lasers are readily able to produce a range of wavelengths through frequency multiplication, with sufficient power to saturate a transition for an ionic ensemble in the trapping region. Schemes are chosen where a dominant decay branch exists to a specific metastable state, from which a more desirable transition may be studied than those from the ground state.

Such an approach was used to avoid $J_{1}=0 \rightarrow J_{\mathrm{u}}=1$ ground-state transitions in order to determine the nuclear spin of ${ }^{100 \mathrm{~m}} \mathrm{Y}$ [23] and in niobium isotopes [5], where in the latter case the ground-state line (used for optical pumping) was additionally too weak for efficient collinear laser spectroscopy. For manganese, the optical pumping scheme utilized a ground-state line too weak for spectroscopy (but nevertheless could be saturated in the cooler-buncher), providing access to a more favorable line in terms of spectroscopic efficiency and sensitivity to the nuclear electric quadrupole moment [18,33]. Spectroscopy of neutralized manganese atoms was also performed by passing the ionic beam through an alkali vapor prior to laser interaction, but the high density of atomic states populated led to an order-of-magnitude loss in efficiency [33]. However, as a consequence, isotope shifts on two lines were therefore measured and MCDF calculations could be 


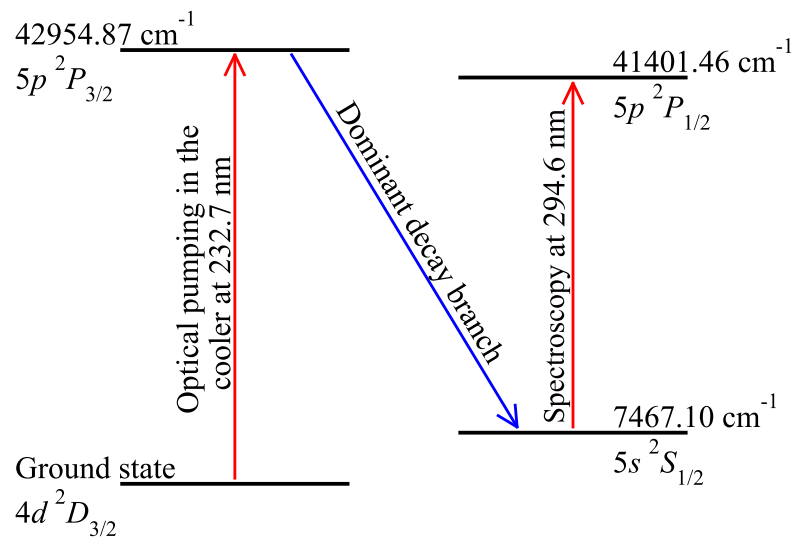

FIG. 2. The optical pumping scheme used to study the 294.6-nm transition in the $\mathrm{Y}^{2+}$ ion.

performed for both lines, although an empirical adjustment had to be made to the mass shift factor for the radii to match regional systematics [19]. Likewise for yttrium, having isotope shifts measured for two lines offers a consistency check of two MCDF calculations, but these were found to be inconsistent, both with each other and the empirical estimates based on nuclear radii systematics [22].

A computationally simpler transition was therefore sought and the 294.6-nm $5 s^{2} S_{1 / 2} \rightarrow 5 p^{2} P_{1 / 2}$ line in the doubly charged ion identified, prompting such spectroscopy to be performed. Since this excitation is from a metastable state at $7467.1 \mathrm{~cm}^{-1}$, optical pumping with $232.7-\mathrm{nm}$ light from a frequency-quadrupled pulsed titanium sapphire laser with a $10-\mathrm{kHz}$ repetition rate was essential to populate this state. The scheme is shown in Fig. 2.

\section{Collinear laser spectroscopy}

After being cooled and bunched, the ions were directed to the collinear laser spectroscopy station. Here, they were overlapped in a collinear geometry with $0.7 \mathrm{~mW}$ of linearly polarized light from a narrow-linewidth (few $\mathrm{MHz}$ ), single-mode, frequency-doubled $\mathrm{cw}$ dye laser, running with Rhodamine-6G dye. The fundamental frequency of the laser was locked to the tip of a Doppler-broadened $\mathrm{I}_{2}$ absorption peak at $16947.1292 \mathrm{~cm}^{-1}$ and stabilized to within a few MHz. A tuning voltage was applied to the laser-ion interaction region to Doppler shift the effective frequency, as seen by the ions over the hyperfine resonances. Ions were resonantly excited on the 294.6-nm $5 s^{2} S_{1 / 2} \rightarrow 5 p^{2} P_{1 / 2}$ transition and fluorescence photons from subsequent decay detected using a photomultiplier tube. To suppress the photon background occurring mainly due to laser light being scattered into the photomultiplier tube, for each tuning voltage step, ions were accumulated in the cooler for $200 \mathrm{~ms}$ and released as a bunch having a $10-\mu$ s temporal length. A gate was applied to the photomultiplier tube signal to veto any photon event occurring without an ion bunch being in front of the detector. By employing ion-beam bunching, the photon background was reduced by 4 orders of magnitude, while preserving the signal.

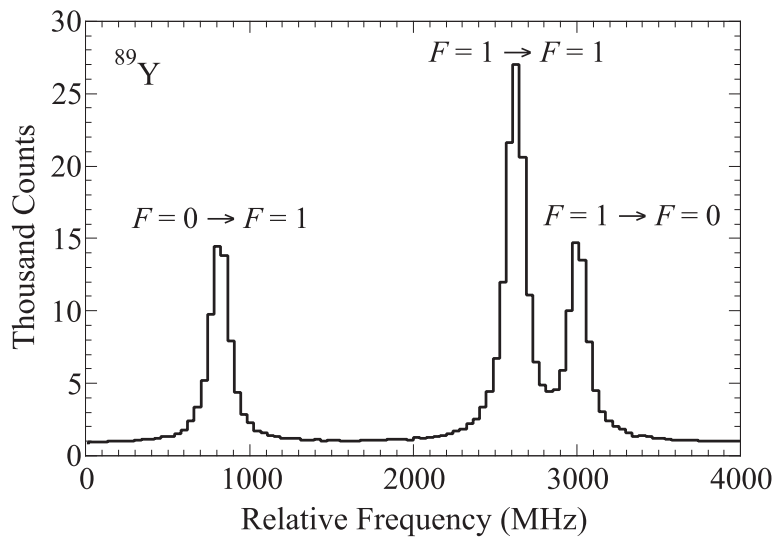

FIG. 3. The three hyperfine peaks of the ${ }^{89} \mathrm{Y}^{2+}(I=1 / 2)$ ion, measured on the 294.6-nm $5 s^{2} S_{1 / 2} \rightarrow 5 p^{2} P_{1 / 2}$ line.

\section{E. Production of the stable yttrium isotope}

Testing of the spectroscopic scheme was performed by forming an ion beam of the stable ${ }^{89} \mathrm{Y}$ isotope. A smaller gas cell was used in place of the fission ion guide, with a few mbar of helium gas, and a pair of electrodes spaced a few millimeters apart. A discharge voltage of $500 \mathrm{~V}$ caused sputtering of a sample of yttrium placed as the cathode. Again, approximately $10 \%$ of the yttrium ions leaving the gas cell were in the doubly charged state.

During radioactive beam experiments, a foil of stable material is normally placed to slightly overlap the entrance aperture of the SPIG [18]. This has the effect of producing a merged beam of the stable isotope which may be measured between scans of radioactive isotopes in order to provide a frequency reference. However, during a first radioactive beam run, it was found that while this method indeed produces ions from the foil material in the singly charged state, it did not produce ions in the doubly charged state (which only originate from within the gas cell).

A dedicated off-line ion source was therefore developed, with a similar arrangement for sputtering in a gas cell as described above. As shown in Fig. 1, this is located separate from the IGISOL chamber providing the radioactive ions. An electrostatic deflector before the analyzer magnet enabled the beam to be accepted from either the IGISOL or the off-line source. To preserve the precise voltage of the cooler, the high voltages of the two sources were both optimized with respect to this.

\section{DATA ANALYSIS}

Ion-beam energies $2 e V_{\text {total }}=2 e\left(V_{\text {cooler }}-V_{\text {tuning }}\right)$ were converted to Doppler-shifted frequencies $v$ by

$$
v=v_{\mathrm{L}}\left(1+\alpha+\sqrt{2 \alpha+\alpha^{2}}\right),
$$

where $v_{\mathrm{L}}$ is the laser frequency in the laboratory frame and $\alpha=$ $2 e V_{\text {total }} / m c^{2}$. An arbitrary reference frequency was subtracted as only relative frequencies are important.

A $\chi$-squared minimization routine was used to fit Lorentzian peaks to the data. For the ${ }^{89} \mathrm{Y}$ spectrum (see example shown in Fig. 3), an ambiguity arises in associating each splitting with the respective upper or lower atomic state, 
TABLE I. Measured hyperfine $A$ parameters for the levels of the 294.6-nm $5 s^{2} S_{1 / 2} \rightarrow 5 p^{2} P_{1 / 2}$ transition in ${ }^{89} \mathrm{Y}^{2+}$, together with theoretical estimates.

\begin{tabular}{lll}
\hline \hline & $A_{\mathrm{l}}(\mathrm{MHz})$ & $A_{\mathrm{u}}(\mathrm{MHz})$ \\
\hline Experimental & $-1803(5)$ & $-391(5)$ \\
Theoretical & -1780 & -352 \\
\hline \hline
\end{tabular}

due to them having an identical $J$. The fitting routine gave values of $A_{1}=-1803(5) \mathrm{MHz}$ and $A_{\mathrm{u}}=-391(5) \mathrm{MHz}$, or equivalently, $A_{1}=+391(5) \mathrm{MHz}$ and $A_{\mathrm{u}}=+1803(5) \mathrm{MHz}$. Although the centroid frequencies (and therefore any isotope shifts extracted) are identical, MCDF calculations of the hyperfine $A$ parameters were performed in order to identify the correct order. Calculated values of $A_{1}=-1780 \mathrm{MHz}$ for the $5 s^{2} S_{1 / 2}$ level and $A_{\mathrm{u}}=-352 \mathrm{MHz}$ for the $5 p^{2} P_{1 / 2}$ level confirm the former option. These results are summarized in Table I. Since $J_{\mathrm{u}, 1}<1$, there is no quadrupole interaction for this line.

During a first radioactive beam experiment, spectra were taken of ${ }^{96,97,98} \mathrm{Y}$ on the 294.6-nm line, shown in Fig. 4. These were chosen as they lie at the peak of fission production and ${ }^{96,98} \mathrm{Y}$, each having nuclear ground-state spins of zero, presenting single-component resonances. While the resonances of ${ }^{96,98} \mathrm{Y}$ could be located and the isotope shift measured, ${ }^{89} \mathrm{Y}^{2+}$ ions were not available to provide a frequency reference. For ${ }^{97} \mathrm{Y}$, one peak was measured, assumed (and later proven) to

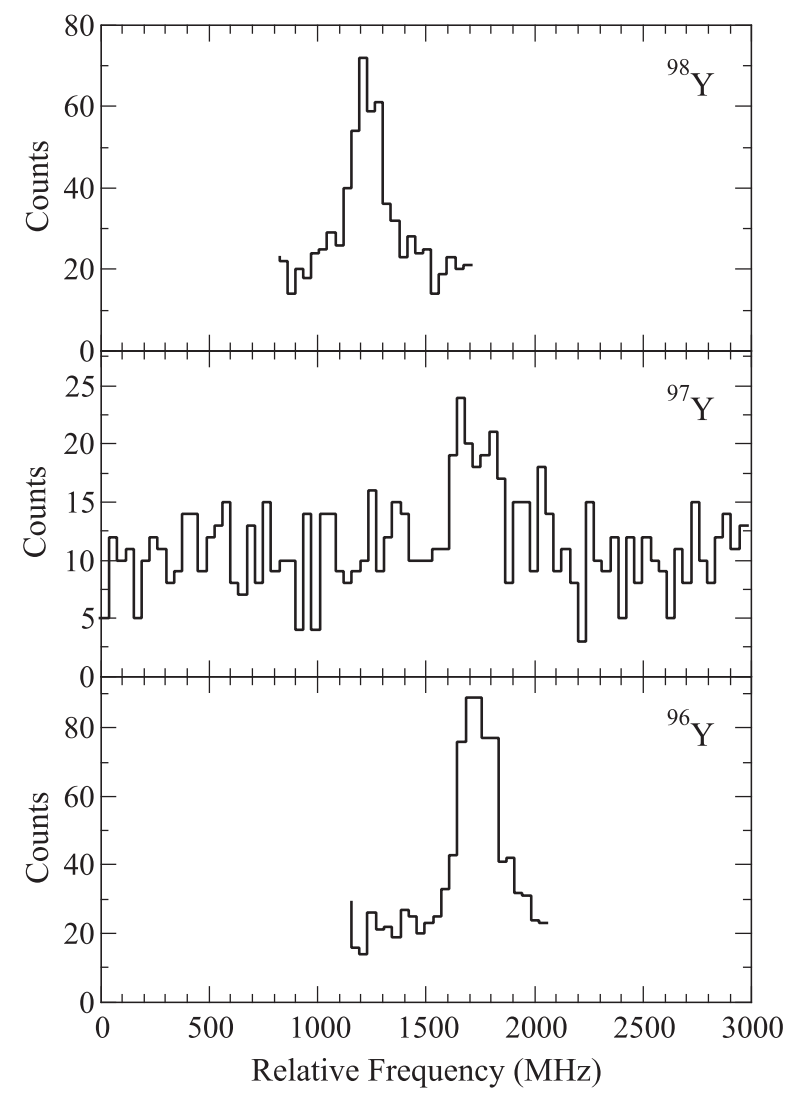

FIG. 4. Summed optical spectra of ${ }^{96,97,98}$ Y taken on the 294.6-nm $\mathrm{Y}^{2+}$ transition during a first experiment.

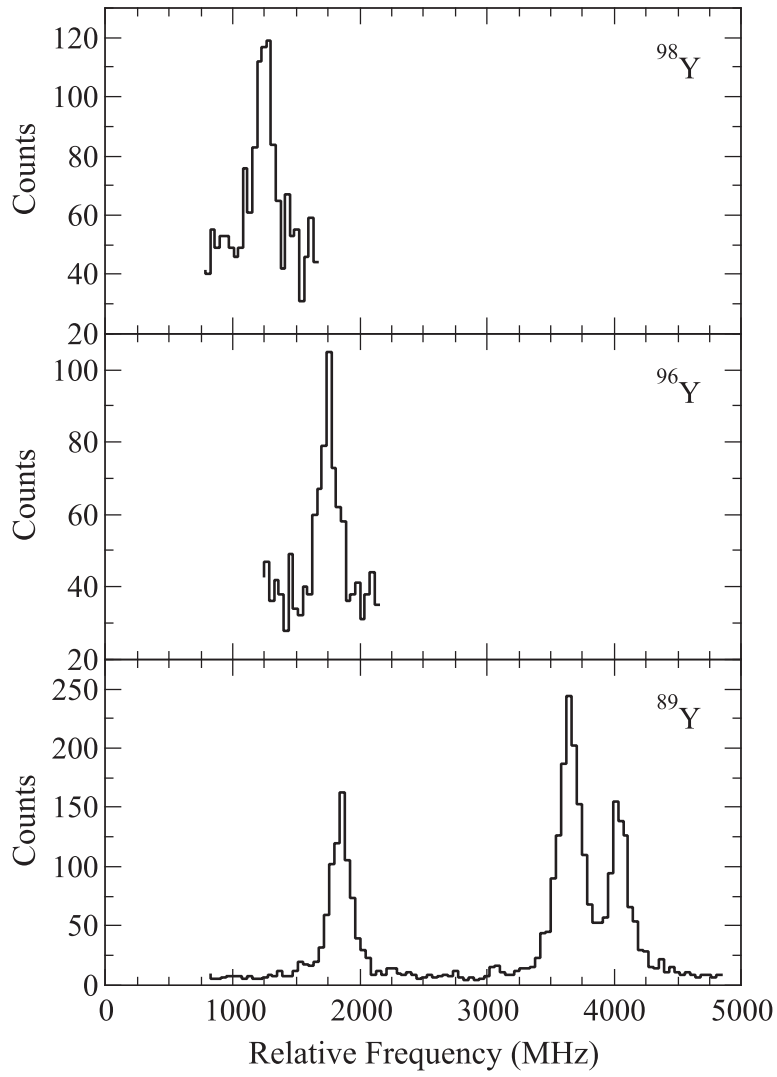

FIG. 5. Summed optical spectra of ${ }^{89,96,98} \mathrm{Y}$ taken on the 294.6-nm $\mathrm{Y}^{2+}$ transition during a second experiment.

be the most intense peak of the ground-state structure. Due to the additional error in determining the centroid of this structure and the close proximity of the modified isotope shift, this could not contribute to making a precise King plot.

Since a minimum of two isotope shifts are required for a King plot, a second experiment was conducted to remeasure ${ }^{96,98} \mathrm{Y}$, but with ${ }^{89} \mathrm{Y}$ ions available simultaneously to provide an accurate frequency reference. Although these spectra had been measured previously at different times, drifts in experimental conditions such as the laser frequency and acceleration voltage can take place between beam times. Measuring all three spectra within a single experiment, shown in Fig. 5, was crucial in minimizing systematic errors for the frequency differences. The isotope shifts measured on the 294.6-nm line are summarized in Table II.

TABLE II. Isotope shifts for ${ }^{89,96,98} \mathrm{Y}$ measured on the 294.6-nm transition, together with the corresponding changes in mean-square charge radii using MCDF calculations of $F$ and $M$ for this transition. Note that the value given for the $\delta v^{96,98}$ isotope shift is from a measurement taken independently of those for $\delta v^{89,96}$ and $\delta v^{89,98}$.

\begin{tabular}{llcc}
\hline \hline$A$ & $A^{\prime}$ & $\delta v^{A, A^{\prime}}(\mathrm{MHz})$ & $\delta\left\langle r^{2}\right\rangle^{A, A^{\prime}}\left(\mathrm{fm}^{2}\right)$ \\
\hline 89 & 96 & $-1546(7)$ & 0.842 \\
89 & 98 & $-2054(8)$ & 1.114 \\
96 & 98 & $-512(9)$ & 0.275 \\
\hline \hline
\end{tabular}




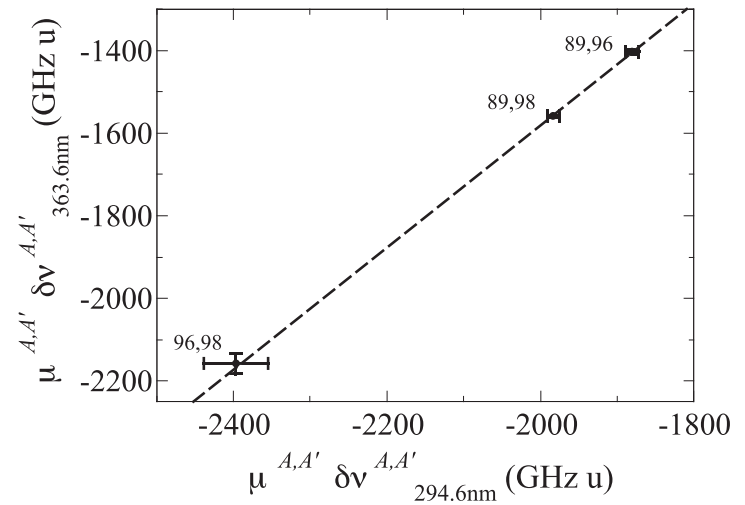

FIG. 6. The King plot of the modified isotope shifts for the 363.3-nm transition used previously [14] and the 294.6-nm transition in the doubly charged ion used in this work.

The isotope shifts for both the 363.3- and 294.6-nm transitions were multiplied by a modification factor

$$
\mu^{A, A^{\prime}}=\frac{m_{A} m_{A^{\prime}}}{m_{A^{\prime}}-m_{A}} .
$$

A King plot was then formed of the modified isotope shifts for the 363.3-nm transition against those for the 294.6-nm transition (Fig. 6). This plot took the form of a straight line with gradient

$$
\frac{F_{363.3 \mathrm{~nm}}}{F_{294.6 \mathrm{~nm}}}=1.480(31)
$$

and intercept

$$
M_{363.3 \mathrm{~nm}}-M_{294.6 \mathrm{~nm}} \frac{F_{363.3 \mathrm{~nm}}}{F_{294.6 \mathrm{~nm}}}=1379(59) \mathrm{GHz} \text { u. }
$$

\section{THEORETICAL CALCULATIONS}

Extensive MCDF calculations were performed to obtain accurate $F$ and $M$ values for the isotope shift of the 294.6$\mathrm{nm} 5 s^{2} S_{1 / 2} \rightarrow 5 p^{2} P_{1 / 2}$ transition in doubly charged yttrium. The atomic wave functions were created using the GRASP $2 \mathrm{~K}$ package [34]. The mass shift parameter $M$ was calculated by evaluating the expectation value of the nuclear recoil operator in the generated atomic wave functions (2) using the RIS3 program [35]. The field-shift parameter $F$ was obtained by calculating the transition energy for several different model nuclei.

In order to monitor convergence, a systematically enlarged multiconfiguration basis is generated by virtual excitations from a set of reference configurations to a maximum of seven layers of correlation orbitals.

The set of reference configurations from which the initial reference basis was built is shown in Table III. It includes the $5 s_{1 / 2}, 5 p_{1 / 2}$, as well as the $5 p_{3 / 2}$ configuration state functions, together with several higher-excited CSFs of the same total angular momentum $J$ and parity $\pi$. After generating wave functions for this reference set (optimizing the three lowest levels for each $J$ and $\pi$ ), the CSF set was reduced to include only those CSFs with a share of at least 0.001 of the $5 s^{2} S_{1 / 2}$ or $5 p^{2} P_{1 / 2}$ level. This yields a set of $18 \mathrm{CSF}$ s of the configurations $4 p^{6} 5 l, 4 p^{5} 4 d 5 l$ and $4 p^{4} 4 d^{2} 5 l(l=s, p)$, which was used
TABLE III. Reference configurations which were used to create the initial estimate for the wave-function basis. In total, these configurations amount to 578 CSFs.

\begin{tabular}{cc}
\hline \hline$J^{\pi}=1 / 2^{+}$ & $J^{\pi}=1 / 2^{-}, 3 / 2^{-}$ \\
\hline$[\mathrm{Ar}] 3 d^{10} 4 s^{2} 4 p^{6} 5 s$ & {$[\mathrm{Ar}] 3 d^{10} 4 s^{2} 4 p^{6} 5 p$} \\
$4 p^{4} 4 d^{2} 5 s$ & $4 p^{4} 4 d^{2} 5 p$ \\
$4 p^{5} 4 d 5 p$ & $4 p^{5} 4 d 5 s$ \\
$4 p^{4} 4 f^{2} 5 s$ & $4 p^{4} 4 f^{2} 5 p$ \\
$4 p^{5} 5 s 5 p$ & $4 p^{5} 5 p^{2}$ \\
$4 p^{4} 5 s 5 p^{2}$ & $4 p^{4} 5 p^{3}$ \\
$4 p^{6} 6 s$ & \\
\hline
\end{tabular}

as the reference for the systematically enlarged basis. This approach of an active space expansion using a multireference has been previously described in Refs. [36,37].

Based on this reference set, single and double excitations from the $4 p, 4 d, 5 s$, and $5 p$ orbitals to correlation orbitals $n l$ with $l \leqslant g$ were considered. Only CSFs which couple to at least one of the reference CSFs (via the Dirac-Coulomb Hamiltonian) were included. This yields basis sets of up to 600,000 CSFs for seven correlation layers, as shown in Table IV. By adding correlation layers, the calculated transition energy converges to a value of $33,850 \mathrm{~cm}^{-1}$, which is within $0.25 \%$ of the experimental value of $33,934 \mathrm{~cm}^{-1}$ [25]. Based on the generated atomic wave functions, the mass and fieldshift parameters, $M$ and $F$, were calculated. The field-shift parameter was calculated by considering model nuclei with mass numbers $A=89,91,93,96,98$.

Table V gives the calculations for $F$ and $M$ (the sum of "normal" (NMS) and "specific" (SMS) mass-shift components) which are seen to converge to

$$
\begin{gathered}
F_{294.6 \mathrm{~nm}}=-1964 \mathrm{MHz} \mathrm{fm}^{-2}, \\
M_{294.6 \mathrm{~nm}}=+130 \mathrm{GHz} \mathrm{u} .
\end{gathered}
$$

A more detailed description of the methods employed here can be found in previous work $[22,36]$.

\section{DISCUSSION}

Equation (1) was used, together with the values of $F$ and $M$, for the 294.6-nm transition calculated via the MCDF method, to extract values of $\delta\left\langle r^{2}\right\rangle$ for ${ }^{89,96} \mathrm{Y}$ and ${ }^{89,98} \mathrm{Y}$, shown in

TABLE IV. Number of CSFs for each number of correlation layers.

\begin{tabular}{lc}
\hline \hline Layers & CSFs \\
\hline 0 & 18 \\
1 & 26,849 \\
2 & 70,541 \\
3 & 135,010 \\
4 & 220,256 \\
5 & 326,279 \\
6 & 453,079 \\
7 & 600,656 \\
\hline \hline
\end{tabular}


TABLE V. Transition energy $\Delta E$ and isotope shift parameters NMS, SMS, and $F$ for each number of correlation layers.

\begin{tabular}{lcccc}
\hline \hline & & \multicolumn{2}{c}{$M(\mathrm{GHzu})$} & \\
\cline { 3 - 4 } Layers & $\Delta E\left(\mathrm{~cm}^{-1}\right)$ & $\mathrm{NMS}$ & $\mathrm{SMS}$ & $F\left(\mathrm{MHz} \mathrm{fm}^{-2}\right)$ \\
\hline 0 & 33,383 & +998 & -958 & -1925 \\
1 & 33,735 & +363 & -235 & -1953 \\
2 & 33,863 & +481 & -325 & -1951 \\
3 & 33,851 & +443 & -333 & -1964 \\
4 & 33,853 & +470 & -352 & -1960 \\
5 & 33,851 & +467 & -345 & -1964 \\
6 & 33,850 & +475 & -346 & -1962 \\
7 & 33,850 & +477 & -347 & -1964 \\
\hline \hline
\end{tabular}

Table II. Moreover, a recalibration of the atomic factors for the 363.3-nm line, and therefore all previously measured radii, could now be done using the King plot. Equations (7)-(10) give the values

$$
\begin{gathered}
F_{363.3 \mathrm{~nm}}=-2908 \mathrm{MHz} \mathrm{fm}^{-2}, \\
M_{363.3 \mathrm{~nm}}=+1571 \mathrm{GHzu} .
\end{gathered}
$$

The recalibrated radii are shown in Fig. 7, together with the empirical estimates [14] and those deduced using MCDF calculations performed for the 363.3- and 321.7-nm lines [22].

Unlike the calculations for the singly charged ion, the calculations for the 294.6-nm line are relatively simple because the levels involved in the transitions have no open $d$ subshells. We believe the calculated value for $F_{294.6 \mathrm{~nm}}$ to be the most precise. However, the value for $M_{294.6 \mathrm{~nm}}$ likely has a larger uncertainty due to the opposing and near-canceling contributions of the NMS and SMS. Nevertheless, it is indeed the charge radii extracted using the atomic factors calculated for the doubly charged ion that provide the closest match to the empirical estimates. It is encouraging that the MCDF calculated factors for the 294.6-nm line yield such similar radii when compared with the empirical estimates, giving added confidence to these values. The only perceptible difference is that the new calculations for the radii after the shape change are a little higher than previous estimates. This confirms yttrium as the isotope chain where the increase in deformation is maximized, during a transition from soft oblate shapes to rigid prolate shapes.

The configurations involved in the $363.3-\mathrm{nm}$ transition are more complicated, although a larger mass shift indicates that this transition at least benefits from a lesser cancellation of the SMS with the NMS. Using calculations for the 321.7-nm line, the agreement of the extracted radii is the poorest. This may be due to a combination of the open $d$ subshell in addition to partial cancellation of the NMS and SMS, where relativistic

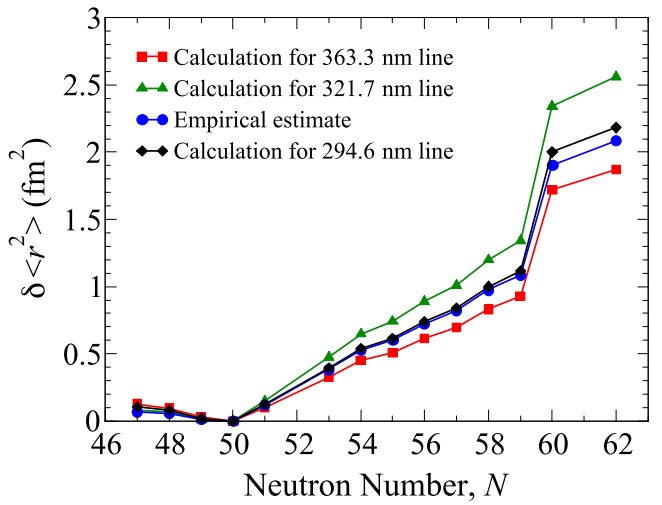

FIG. 7. Mean-square charge radii of yttrium isotopes calculated using an empirical estimate [14] and MCDF calculations for the 363.3-nm line [22], the 321.7-nm line [22], and the 294.6-nm line of this work.

effects (neglected in earlier calculations) may play a significant role.

\section{SUMMARY}

Isotope shifts of selected isotopes of yttrium have been remeasured using a different transition to two previously studied in order to assess the reliability of MCDF calculations, and to provide confidence in the nuclear mean-square charge radii values previously extracted. In this case the chosen transition is in the doubly charged ion and removes the complication of an open $d$ subshell in the calculations, which provides radii closely matching earlier (empirical) estimates. We encourage further developments in such calculations, which are crucial for elements where a calibration for the radii does not exist due to there being too few naturally occurring isotopes.

For such studies, the IGISOL IV facility possesses versatility in terms of production, which is fast and universal, irrespective of chemical or physical properties. Among other purposes, optical pumping enables the study of a greater range of transitions, not having the ground state in common, to better benchmark MCDF calculations. The availability of doubly charged ions gives further flexibility, and an alkali vapor charge exchange cell [38] will soon be installed immediately upstream of the photon detection region for studies of neutralized atoms.

\section{ACKNOWLEDGMENTS}

This work has been supported by the UK Science and Technology Facilities Council (STFC), the Academy of Finland, under the Finnish Centre of Excellence Programme 20122017 (Project No. 251353), Nuclear and Accelerator Based Physics Research at JYFL, and the European Union Horizon 2020 research and innovation program under Grant Agreement No. 654002 (ENSAR2). R.B., S.F., and S.O.S. acknowledge support by the German Federal Ministry of Education and Research (BMBF) under Contract No. 05P15SJCIA.
[1] P. Campbell, I. D. Moore, and M. R. Pearson, Prog. Part. Nucl. Phys. 86, 127 (2016).
[2] K. Blaum, J. Dilling, and W. Nörtershäuser, Phys. Scr. T 152, 014017 (2013). 
[3] B. Cheal and K. T. Flanagan, J. Phys. G: Nucl. Part. Phys. 37, 113101 (2010).

[4] R. Neugart, J. Billowes, M. L. Bissell, K. Blaum, B. Cheal, K. T. Flanagan, G. Neyens, W. Nörtershäuser, and D. T. Yordanov, J. Phys. G: Nucl. Part. Phys. 44, 064002 (2017).

[5] B. Cheal, K. Baczynska, J. Billowes, P. Campbell, F. C. Charlwood, T. Eronen, D. H. Forest, A. Jokinen, T. Kessler, I. D. Moore et al., Phys. Rev. Lett. 102, 222501 (2009).

[6] K. Minamisono, D. M. Rossi, R. Beerwerth, S. Fritzsche, D. Garand, A. Klose, Y. Liu, B. Maaß, P. F. Mantica, A. J. Miller et al., Phys. Rev. Lett. 117, 252501 (2016).

[7] A. Voss, M. R. Pearson, J. Billowes, F. Buchinger, B. Cheal, J. E. Crawford, A. A. Kwiatkowski, C. D. Philip Levy, and O. Shelbaya, Phys. Rev. Lett. 111, 122501 (2013).

[8] V. Fedosseev, K. Chrysalidis, T. D. Goodacre, B. Marsh, S. Rothe, C. Seiffert, and K. Wendt, J. Phys. G 44, 084006 (2017).

[9] M. Laatiaoui, W. Lauth, H. Backe, M. Block, D. Ackermann, B. Cheal, P. Chhetri, C. E. Düllmann, P. van Duppen, J. Even et al., Nature (London) 538, 495 (2016).

[10] M. Wang, G. Audi, F. G. Kondev, W. J. Huang, S. Naimi, and X. Xu, Chin. Phys. C 41, 030003 (2017).

[11] G. Fricke, C. Bernhardt, K. Heilig, L. A. Schaller, L. Schellenberg, E. B. Shera, and C. W. Dejager, At. Data Nucl. Data Tables 60, 177 (1995).

[12] I. Angeli and K. Marinova, At. Data Nucl. Data Tables 99, 69 (2013).

[13] K. Tsukada, A. Enokizono, T. Ohnishi, K. Adachi, T. Fujita, M. Hara, M. Hori, T. Hori, S. Ichikawa, K. Kurita et al. Phys. Rev. Lett. 118, 262501 (2017).

[14] B. Cheal, M. D. Gardner, M. Avgoulea, J. Billowes, M. L. Bissell, P. Campbell, T. Eronen, K. T. Flanagan, D. H. Forest, J. Huikari et al., Phys. Lett. B 645, 133 (2007).

[15] P. Campbell, H. L. Thayer, J. Billowes, P. Dendooven, K. T. Flanagan, D. H. Forest, J. A. R. Griffith, J. Huikari, A. Jokinen, R. Moore et al., Phys. Rev. Lett. 89, 082501 (2002).

[16] F. C. Charlwood, K. Baczynska, J. Billowes, P. Campbell, B. Cheal, T. Eronen, D. H. Forest, A. Jokinen, T. Kessler, I. D. Moore et al., Phys. Lett. B 674, 23 (2009).

[17] M. Avgoulea, Yu. P. Gangrsky, K. P. Marinova, S. G. Zemlyanoi, S. Fritzsche, D. Iablonskyi, C. Barbieri, E. C. Simpson, P. D. Stevenson, J. Billowes et al., J. Phys. G: Nucl. Part. Phys. 38, 025104 (2011).

[18] F. C. Charlwood, J. Billowes, P. Campbell, B. Cheal, T. Eronen, D. H. Forest, S. Fritzsche, M. Honma, A. Jokinen, I. D. Moore et al., Phys. Lett. B 690, 346 (2010).
[19] H. Heylen et al., Phys. Rev. C 94, 054321 (2016).

[20] M. L. Bissell, T. Carette, K. T. Flanagan, P. Vingerhoets, J. Billowes, K. Blaum, B. Cheal, S. Fritzsche, M. Godefroid, M. Kowalska et al., Phys. Rev. C 93, 064318 (2016).

[21] T. J. Procter et al., Phys. Rev. C 86, 034329 (2012).

[22] B. Cheal, T. E. Cocolios, and S. Fritzsche, Phys. Rev. A 86, 042501 (2012).

[23] K. Baczynska, J. Billowes, P. Campbell, F. C. Charlwood, B. Cheal, T. Eronen, D. H. Forest, A. Jokinen, T. Kessler, I. D. Moore et al., J. Phys. G: Nucl. Part. Phys. 37, 105103 (2010).

[24] M. L. Bissell, K. T. Flanagan, M. D. Gardner, M. Avgoulea, J. Billowes, P. Campbell, B. Cheal, T. Eronen, D. H. Forest, J. Huikari et al., Phys. Lett. B 645, 330 (2007).

[25] G. L. Epstein and J. Reader, J. Opt. Soc. Am. 65, 310 (1975).

[26] B. Cheal and D. H. Forest, Hyperfine Interact. 223, 63 (2014).

[27] D. H. Forest and B. Cheal, Hyperfine Interact. 223, 207 (2014).

[28] B. Cheal and D. H. Forest, Hyperfine Interact. 223, 223 (2014).

[29] J. M. G. Levins, D. M. Benton, J. Billowes, P. Campbell, T. G. Cooper, P. Dendooven, D. E. Evans, D. H. Forest, I. S. Grant, J. A. R. Griffith et al., Phys. Rev. Lett. 82, 2476 (1999).

[30] I. D. Moore, P. Dendooven, and J. Ärje, Hyperfine Interact., 223 17 (2014).

[31] P. Karvonen, I. D. Moore, T. Sonoda, T. Kessler, H. Penttilä, K. Peräjärvi, P. Ronkanen, and J. Äystö, Nucl. Instrum. Methods Phys. Res., Sect. B 266, 4794 (2008).

[32] A. Nieminen, P. Campbell, J. Billowes, D. H. Forest, J. A. R. Griffith, J. Huikari, A. Jokinen, I. D. Moore, R. Moore, G. Tungate, J. Aysto et al., Phys. Rev. Lett. 88, 094801 (2002).

[33] C. Babcock, H. Heylen, M. L. Bissell, K. Blaum, P. Campbell, B. Cheal, D. Fedorov, R. F. Garcia-Ruiz, W. Geithner, W. Gins et al., Phys. Lett. B 760, 387 (2016).

[34] P. Jönsson, G. Gaigalas, J. Bieroń, C. F. Fischer, and I. P. Grant, Comput. Phys. Commun. 184, 2197 (2013).

[35] C. Nazé et al., Comput. Phys. Commun. 184, 2187 (2013).

[36] L. Filippin, R. Beerwerth, J. Ikman, S. Fritzsche, M. Godefroid, and P. Jönsson, Phys. Rev. A 94, 062508 (2016).

[37] L. Filippin, M. Godefroid, J. Ekman, and P. Jönsson, Phys. Rev. A, 93, 062512 (2016).

[38] A. Klose, K. Minamisono, Ch. Geppert, N. Frömmgen, M. Hammen, J. Krämer, A. Krieger, C. D. P. Levy, P. F. Mantica, W. Nörtershäuser, and S. Vinnikova, Nucl. Instrum. Methods Phys. Res., Sect. A 678, 114 (2012). 